\title{
Reprodutibilidade de um protocolo de esforço durante a corrida em piscina funda
}

\author{
Reproducibility of an effort protocol during deep-water running
}

\author{
I.R.S. Silva, L.S. Oliveira, M.F. Berenguer, A.V.F. Sousa, J.A. Nascimento, M.C. Costa
} \begin{abstract}
Diversos protocolos são utilizados a fim de obter o esforço máximo por meio da corrida em piscina funda (DWR). Entretanto, poucos estudos analisaram a validade e reprodutibilidade em testes incrementais com a DWR. Assim, o objetivo deste estudo foi verificar a reprodutibilidade interavaliador de um protocolo durante a corrida em piscina funda. Em uma pesquisa comparativa e correlacional, 20 universitários fisicamente ativos $(21.10 \pm 1.68$ anos, $68.90 \pm 7.24 \mathrm{~kg}, 174.60 \pm 5.35$ $\mathrm{cm}, \Sigma 7 \mathrm{DC} 106.80 \pm 28.63 \mathrm{~mm}$ ) foram avaliados por dois examinadores diferentes em um protocolo submáximo de corrida em piscina funda com intensidade inicial de 100 ciclos por minuto e incremento de quatro a cada minuto. Medidas de FC e PSE foram registadas a cada estágio. A reprodutibilidade foi analisada pelo coeficiente de correlação de Pearson $(r)$ e intra-classe (CCI), e teste de Wilcoxon. Não houve diferença significativa entre as medições dos examinadores para FC de repouso $(p>.05)$, inicial $(p>.05)$ e de recuperação $(p>.05)$. Apesar das diferenças significativas $(p<.05)$ encontradas para a PSE nos estágios E1, E5, E7, E8 e E9, verificaram-se boas correlações significativas $\left(r>.60, \mathrm{CCI}_{95 \%}>\right.$ .60) para as medidas dessas variáveis inter-avaliador. Os resultados mostraram boa reprodutibilidade inter-avaliador para as variáveis de FC e PSE em diferentes cadências, indicando que o protocolo possui aplicação profissional.
\end{abstract}

Palavras-chave: exercício, esforço físico, psicometria

ABSTRACT

The aim of this study was to verify the inter-assessment reproducibility of heart rate (HR) and rating of perceived exertion (RPE) in exercise with different cadences during deep water running. In a comparative and correlational study, twenty physically active college students (aged $21.10 \pm 1.68 \mathrm{yr}$, body mass $68.90 \pm 7.24 \mathrm{~kg}$, height $174.60 \pm 5.35 \mathrm{~cm}$, $\Sigma 7 \mathrm{SF} 106.80 \pm 28.63 \mathrm{~mm}$ ) were assessed twice by two different evaluators in a 12-stage sub maximal protocol of deep water running at initial intensity of 100 cycles per minute with an increment of four cycle per minute (E1 - E12). HR and RPE measures were registered at each stage. Reproducibility was analyzed by Pearson linear coefficient $(r)$ and intraclass correlation (ICC), and Wilcoxon test. No significant differences were found among evaluators' measures for the resting $(p>.05)$, basal $(p>.05)$ and recovery $(p>.05)$ heart rate. Significant differences $(p<.05)$ were found for RPE in E1, E5, E7, E8 and E9 stages. Inter-assessment correlations were significant $\left(r>.60, \mathrm{ICC}_{95 \%}>.60\right)$. The results show good intra-assessment reproducibility for HR and RPE at different cadences, indicating that the protocol has professional application.

Keywords: exercise, physical effort, psychometrics

Submetido: 11.12.2009 | Aceite: 29.06.2010

Iana Rafaella Santiago da Silva. Grupo de Pesquisa em Avaliação da Performance Humana - ESEF/UPE; Bolsista CAPES, Brasil.

Leonardo dos Santos Oliveira. Grupo de Pesquisa em Avaliação da Performance Humana - ESEF/UPE; Bolsista do Instituto de Apoio a Universidade de Pernambuco - IAUPE, Brasil.

Mariana de Freitas Berenguer. Grupo de Pesquisa em Avaliação da Performance Humana - ESEF/UPE; Escola Superior de Educação Física da Universidade de Pernambuco; Bolsista PIBIC/FACEPE/CNPq, Brasil.

Arthur Vale Franklin de Sousa e Manoel da Cunha Costa. Grupo de Pesquisa em Avaliação da Performance Humana - ESEF/UPE; Escola Superior de Educação Física da Universidade de Pernambuco, Brasil.

João Agnaldo do Nascimento. Universidade Federal da Paraíba, Brasil.

Endereço para correspondência: Manoel da Cunha Costa, Rua Arnóbio Marques, nº 310, Campus Universitário HUOC - ESEF, CEP: 50.100-130, Santo Amaro, Recife - PE, Brasil.

E-mail: mcosta2@gmail.com 
Os exercícios aquáticos em posição vertical são amplamente utilizados por diversos profissionais da área da saúde, devido aos inúmeros benefícios proporcionados pela água. Atividades físicas na água são comuns na reabilitação e condicionamento físico, em que se pode atestar melhorias do sistema cardiorrespiratório e muscular (Dolbow, Farley, Kim, \& Caputo, 2008). Adicionalmente, verifica-se que há redução do estresse sobre as articulações durante este tipo de esforço físico (Alves, Mota, Costa, \& Alves, 2004).

Entre as atividades mais utilizadas, estudos indicam que a corrida na água em piscina funda, Deep Water Running (DWR), proporciona melhorias das condições patológicas de indivíduos com problemas nas articulações dos membros inferiores (Kilding, Scott, \& Mullineaux, 2007) e massa corporal elevada (McGhee, Power, \& Steele, 2007), de atletas em recuperação de lesões (Pantoja, Vendrusculo, Fayh, Alberton, \& Kruel, 2006), como também para reabilitação cardíaca (Green, Cable, \& Elms, 1990) e treino para evitar o overtraining (Broman, Quintana, Lindberg, Jansson, \& Kaijser, 2006).

No tocante à prescrição do exercício, uma das características mais importantes para o alcance de efeitos benéficos na saúde ou desempenho físico por meio da DWR é o controle da intensidade durante o treino. A Aquatic Exercise Association (Lindle, Wasserman, \& See, 2001) bem como o American College of Sports Medicine (Nelson et al., 2007) recomendam monitorar e prescrever a intensidade dos exercícios com predominância aeróbia por meio da frequência cardíaca (FC) ou da percepção subjetiva do esforço (PSE) (Pollock et al., 1998).

Nesse sentido, diversos protocolos têm sido utilizados a fim de obter o esforço máximo por meio da DWR, com base nas respostas da FC, PSE, consumo máximo de oxigênio e razão de troca respiratória (Mercer \& Jensen, 1997; Michaud, Rodriguez-Zayas, Andres, Flynn, \& Lambert, 1995; Wilder, Brennan, \& Schotte,
1993). No entanto, poucos estudos analisaram a validade e reprodutibilidade (Mercer \& Jensen, 1997) em testes incrementais com a DWR.

Assim, o objetivo deste estudo é verificar a reprodutibilidade interavaliador de um protocolo de esforço durante a corrida em piscina funda.

\section{MÉTODO}

Trata-se de uma pesquisa comparativa e correlacional, com abordagem transversal a partir de dados primários.

\section{Amostra}

A população foi de indivíduos fisicamente ativos (Craig et al., 2003). A amostra foi composta por 20 universitários de 19 a 25 anos de idade, estimada pelo software GPower 3.0, considerando-se um poder mínimo de .80 e $\alpha$ $=.05$. Foram incluídos homens aparentemente saudáveis, com boa adaptação ao meio líquido (flutuar, deslocar e mergulhar), mas sem experiência com a corrida em piscina funda, para que não houvesse interferência em relação ao tempo de prática. Foram excluídos atletas, fumantes ou indivíduos que já praticaram algum tipo de exercício na água em posição vertical e usuários de substâncias com ação cardiovascular.

\section{Instrumentos e Procedimentos}

Antes das avaliações, um único avaliador realizou uma bateria de medidas morfológicas de forma rotacional e padronizadas pela International Society for the Advancement of Kinantropometry (2000). A massa corporal e a estatura foram medidas em balança digital com estadiômetro (Filizola, Brasil) próximo a $.1 \mathrm{~kg}$ e $.1 \mathrm{~cm}$, respectivamente. Medidas de dobras cutâneas foram tomadas em sete locais (subescapular, tríceps, axilar média, abdominal, suprailíaca, coxa e perna) com plicômetro (Lange Skinfold Caliper, Maryland, EUA) próximo a $\quad .1 \mathrm{~mm}$. Os valores considerados para cada local se deram pela média de duas medições consecutivas e que 
estas não poderiam diferir por mais de $5 \%$; nesse caso a mediana dos três valores foi usada. As características físicas dos indivíduos são mostradas na tabela 1 .

Tabela 1

Características físicas da amostra $(n=20)$

\begin{tabular}{lcc}
\hline \multicolumn{1}{c}{ Variável } & M $\pm \mathrm{DP}$ & Min - Máx \\
\hline Idade (anos) & $21.10 \pm 1.68$ & $19.0-25.0$ \\
Massa corporal $(\mathrm{kg})$ & $68.90 \pm 7.24$ & $59.5-90.2$ \\
Estatura $(\mathrm{cm})$ & $174.6 \pm 5.35$ & $165.0-185.0$ \\
IMC $\left(\mathrm{kg} / \mathrm{m}^{2}\right)$ & $22.10 \pm 1.65$ & $19.9-27.5$ \\
$\Sigma 7 D C(\mathrm{~mm})$ & $106.8 \pm 28.63$ & $64.0-168.0$ \\
\hline
\end{tabular}

\section{Protocolo de esforço para a corrida em piscina} funda

Os sujeitos foram avaliados por dois examinadores diferentes (A e B), separados por sete dias, em que cada dia um examinador treinado aplicou o protocolo.

Valores de frequência cardíaca em repouso foram tomados após cinco minutos com o sujeito dentro da piscina sobre uma plataforma, em que era mantido com a cabeça acima da superfície da água.

Realizou-se um aquecimento de dois minutos com uma cadência de 80 ciclos por minuto (cpm). A mensuração da FC (Polar, Finlândia) e PSE (Escala de Borg 6-20) envolveu um protocolo contínuo de corrida em piscina funda a uma intensidade inicial de 100 cpm com incremento de quatro a cada estágio, até a exaustão voluntária ou falha na execução do movimento padronizado. Cada estágio tinha duração de um minuto.

Um colete flutuador (Coretech, Brasil), com capacidade máxima de $120 \mathrm{~kg}$, foi fixado no sujeito para permitir a sua flutuabilidade, sendo preso à borda da piscina para evitar o deslocamento horizontal.

Avisos sonoros (bips) foram reproduzidos para indicar a cadência dos estágios.

Os sujeitos foram instruídos a manter a cadência, alternando movimentos de elevação de joelhos e braços, sendo encorajados verbalmente durante todo o esforço.

\section{Aspectos éticos}

Este estudo foi aprovado pelo Comitê de Ética em Pesquisa da Universidade de Pernambuco (UPE), Brasil, com o registo 166/08. Quanto aos procedimentos inerentes às avaliações, em um momento prévio à solicitação da assinatura do Termo de Consentimento Livre e Esclarecido por parte dos responsáveis, foram explicados os objetivos da pesquisa, bem como informados os possíveis riscos e benefícios do estudo, além da confidencialidade das informações a serem adquiridas.

\section{Análise Estatística}

Dados de variáveis quantitativas foram reportados por média $(M)$ e desvio-padrão $(D P)$ e valores máximos e mínimos. Verificouse a normalidade dos dados (teste de ShapiroWilk) antes das análises. Comparações entre diferentes examinadores foram verificadas em dias distintos (teste pareado de Wilcoxon). Análises de reprodutibilidade foram efetuadas para o teste em piscina funda (coeficiente linear de Pearson e de correlação intra-classe CCI). O nível de significância adotado para todas as análises foi de $p<.05$ (bicaudal). Utilizou-se o pacote SPSS 16.0 (SPSS Inc., Chicago, IL, USA).

\section{RESULTADOS}

Não houve diferença significativa entre as médias de frequência cardíaca de repouso $\left(\mathrm{FC}_{\mathrm{REP}}\right)$, inicial $\left(\mathrm{FC}_{\mathrm{INI}}\right)$ e de recuperação $\left(\mathrm{FC}_{\mathrm{REC}}\right)$ e percepção subjetiva de esforço obtidas pelos examinadores (tabela 2). Foram encontrados valores significativos $(p<.05)$ superiores a .63 para o coeficiente linear de Pearson e acima de .77 para o coeficiente de correlação intra-classe para as medidas dessas variáveis durante a aplicação do protocolo entre os avaliadores (tabela 2).

Durante os estágios do protocolo, as médias de FC e PSE aumentaram gradativamente em relação ao incremento na intensidade, sendo similar nas observações dos avaliadores A e B (tabela 3). 
50 | I.R.S. Silva, L.S. Oliveira, M.F. Berenguer, A.V.F. Sousa, J.A. Nascimento, M.C. Costa

Tabela 2

Medidas descritivas para temperatura, frequências cardíacas (FC), teste de Wilcoxon (p), correlação de Pearson $(r) e$ coeficiente de correlação intra-classe (CCI)

\begin{tabular}{|c|c|c|c|c|c|c|c|}
\hline Variável & Examinador & Mín. & Máx. & $\mathrm{M} \pm \mathrm{DP}$ & $p$ & $r$ & CCI $\left(\mathrm{IC}_{95 \%}\right)$ \\
\hline \multirow{2}{*}{ Temp $\left({ }^{\circ} \mathrm{C}\right)$} & A & 28 & 30 & $29.0 \pm .6$ & \multirow{2}{*}{.152} & \multirow{2}{*}{ - } & \multirow{2}{*}{ - } \\
\hline & B & 28 & 29.5 & $28.8 \pm .5$ & & & \\
\hline \multirow{2}{*}{$\mathrm{FC}_{\mathrm{REP}}(b p m)$} & A & 67 & 112 & $91.2 \pm 14.1$ & \multirow{2}{*}{.056} & \multirow{2}{*}{$.633^{*}$} & \multirow{2}{*}{$.774(.429-.910)$} \\
\hline & $\mathrm{B}$ & 50 & 106 & $84.8 \pm 13.2$ & & & \\
\hline \multirow{2}{*}{$\mathrm{FC}_{\mathrm{INI}}(b p m)$} & A & 99 & 142 & $122.8 \pm 11.8$ & \multirow{2}{*}{.840} & \multirow{2}{*}{$.741^{*}$} & \multirow{2}{*}{$.798(.489-.920)$} \\
\hline & B & 93 & 152 & $123.6 \pm 19.1$ & & & \\
\hline \multirow{2}{*}{$\mathrm{FC}_{\mathrm{REC}}(b p m)$} & A & 115 & 162 & $138.1 \pm 13.3$ & \multirow{2}{*}{.197} & \multirow{2}{*}{$.823^{*}$} & \multirow{2}{*}{$.902(.753-.961)$} \\
\hline & B & 110 & 166 & $135.0 \pm 14.0$ & & & \\
\hline
\end{tabular}

Tabela 3

Medidas descritivas para as variáveis frequências cardíaca (FC), teste de Wilcoxon (p), correlação de Pearson (r) e coeficiente de correlação intra-classe para os examinadores A e B para os estágios E1 a E12

\begin{tabular}{|c|c|c|c|c|c|c|c|c|}
\hline \multirow{2}{*}{ Estágio } & \multicolumn{2}{|c|}{$n$} & \multirow{2}{*}{ Var. } & \multirow{2}{*}{$\begin{array}{c}\text { Examinador } \mathrm{A} \\
\mathrm{M} \pm \mathrm{DP}\end{array}$} & \multirow{2}{*}{$\begin{array}{c}\text { Examinador B } \\
\mathrm{M} \pm \mathrm{DP}\end{array}$} & \multirow{2}{*}{$p$} & \multirow{2}{*}{$r^{*}$} & \multirow{2}{*}{ CCI $\left(\mathrm{IC}_{95 \%}\right)$} \\
\hline & $\mathrm{A}$ & $\mathrm{B}$ & & & & & & \\
\hline \multirow{2}{*}{ E1 } & \multirow{2}{*}{20} & \multirow{2}{*}{20} & $\mathrm{FC}$ & $132.30 \pm 13.66$ & $133.25 \pm 20.55$ & .872 & .727 & $.803(.502, .922)$ \\
\hline & & & PSE & $9.80 \pm 1.47$ & $8.90 \pm 2.00$ & $.024^{*}$ & .279 & $.421(-.463, .771)$ \\
\hline \multirow{2}{*}{ E2 } & \multirow{2}{*}{20} & \multirow{2}{*}{20} & FC & $139.65 \pm 16.03$ & $138.35 \pm 20.88$ & .532 & .771 & $.854(.631, .942)$ \\
\hline & & & PSE & $10.95 \pm 1.47$ & $10.00 \pm 2.29$ & .098 & .359 & $.492(-.283, .799)$ \\
\hline \multirow{2}{*}{ E3 } & \multirow{2}{*}{20} & \multirow{2}{*}{20} & $\mathrm{FC}$ & $146.20 \pm 17.40$ & $143.90 \pm 19.55$ & .076 & .849 & $.915(.786, .966)$ \\
\hline & & & PSE & $12.30 \pm 1.75$ & $11.50 \pm 2.76$ & .121 & .490 & $.614(.025, .847)$ \\
\hline \multirow[b]{2}{*}{ E4 } & \multirow{2}{*}{20} & \multirow{2}{*}{19} & $\mathrm{FC}$ & $151.15 \pm 15.66$ & $147.11 \pm 20.66$ & .088 & .806 & $.869(.659, .949)$ \\
\hline & & & PSE & $13.00 \pm 1.84$ & $11.95 \pm 2.48$ & .063 & .649 & $.765(.390, .910)$ \\
\hline \multirow{2}{*}{ E5 } & \multirow{2}{*}{20} & \multirow{2}{*}{19} & $\mathrm{FC}$ & $158.65 \pm 16.31$ & $153.26 \pm 18.87$ & .070 & .769 & $.863(.644, .947)$ \\
\hline & & & PSE & $14.45 \pm 2.01$ & $13.11 \pm 2.45$ & $.007^{*}$ & .809 & $.883(.697, .955)$ \\
\hline \multirow{2}{*}{ E6 } & \multirow{2}{*}{19} & \multirow{2}{*}{19} & $\mathrm{FC}$ & $162.63 \pm 15.02$ & $158.58 \pm 19.36$ & .099 & .851 & $.903(.750, .963)$ \\
\hline & & & PSE & $15.00 \pm 1.86$ & $14.26 \pm 2.38$ & .081 & .730 & $.830(.557, .934)$ \\
\hline \multirow{2}{*}{ E7 } & \multirow{2}{*}{17} & \multirow{2}{*}{18} & FC & $166.76 \pm 15.50$ & $162.78 \pm 17.08$ & .097 & .837 & $.907(.744, .966)$ \\
\hline & & & PSE & $16.41 \pm 1.87$ & $15.11 \pm 2.40$ & $.005^{*}$ & .706 & $.719(.501, .935)$ \\
\hline \multirow{2}{*}{ E8 } & \multirow{2}{*}{14} & \multirow{2}{*}{17} & $\mathrm{FC}$ & $169.21 \pm 14.97$ & $165.24 \pm 15.51$ & $.026^{*}$ & .888 & $.939(.810, .980)$ \\
\hline & & & PSE & $16.79 \pm 1.80$ & $16.06 \pm 2.33$ & $.012 *$ & .658 & $.790(.347, .933)$ \\
\hline & & & $\mathrm{FC}$ & $170.92 \pm 14.69$ & $168.88 \pm 15.13$ & .065 & .893 & $.939(.788, .982)$ \\
\hline E9 & 12 & 17 & PSE & $17.42 \pm 1.68$ & $17.06 \pm 2.28$ & $.031^{*}$ & .744 & $.840(.445, .954)$ \\
\hline & & & FC & $175.00 \pm 15.46$ & $172.73 \pm 14.38$ & .065 & .872 & $.930(.716, .982)$ \\
\hline E10 & 10 & 15 & PSE & $17.30 \pm 1.57$ & $17.67 \pm 1.99$ & .396 & .745 & $.839(.352, .960)$ \\
\hline & & & $\mathrm{FC}$ & $177.25 \pm 12.94$ & $176.58 \pm 14.90$ & .396 & .812 & $.876(.382, .975)$ \\
\hline Ell & 8 & 12 & PSE & $18.25 \pm 1.17$ & $18.08 \pm 1.88$ & .131 & .548 & $.662(-.690, .932)$ \\
\hline & & & $\mathrm{FC}$ & $180.75 \pm 11.91$ & $177.00 \pm 15.67$ & .205 & .899 & $.919(.584, .984)$ \\
\hline E12 & 8 & 9 & PSE & $18.63 \pm 1.19$ & $18.11 \pm 1.90$ & .098 & .806 & $.842(.209, .968)$ \\
\hline
\end{tabular}

$* p<.05$ 
Destaca-se que o número de sujeitos ( $n$ ) foi inversamente proporcional à intensidade, considerando-se que apenas de 8 a 9 sujeitos completaram o estágio final do teste (ver tabela 3).

O coeficiente linear de Pearson para a medida da PSE entre os examinadores foi baixo para os primeiros estágios, bem como o CCI, mas se estabilizou ao longo dos estágios. Nas medições dos examinadores A e B por estágio, diferenças foram observadas com relação à $\mathrm{FC}$ e PSE em alguns estágios (tabela 3). A partir do estágio $E 5$, ao passo em que a cadência aumentava (principalmente em E7, E8 e E9), observaram-se diferenças significativas, sobretudo em relação à PSE. No entanto, a partir do décimo estágio, não houve diferença nos valores da FC e PSE para os examinadores A e B $(p>.05)$, como apresentado na tabela 3.

Conforme ilustrado pelas figuras 1 e 2 , que apresentam respectivamente os valores médios da FC e PSE medidas durante o protocolo de esforço pelos examinadores A e B, foram obtidos valores similares após a aplicação dos mesmos procedimentos, nas mesmas condições de estudo.

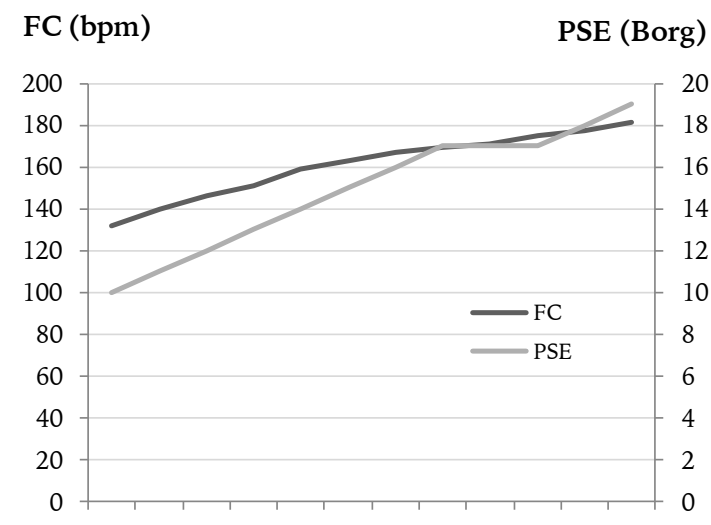

E1 E2 E3 E4 E5 E6 E7 E8 E9 E10E11E12

Figura 1. Médias por estágios da frequência cardíaca

(FC) e percepção de esforço (PSE) obtidas pelo examinador $\mathrm{A}$

Os valores médios da PSE se aproximam dos valores médios da FC na medida em que se avança para novos estágios (incremento da carga). A partir do oitavo estágio, estes valores estão muito próximos, confirmando a alta relação entre estas variáveis. Esse fato foi evidente nas medidas de ambos os avaliadores.

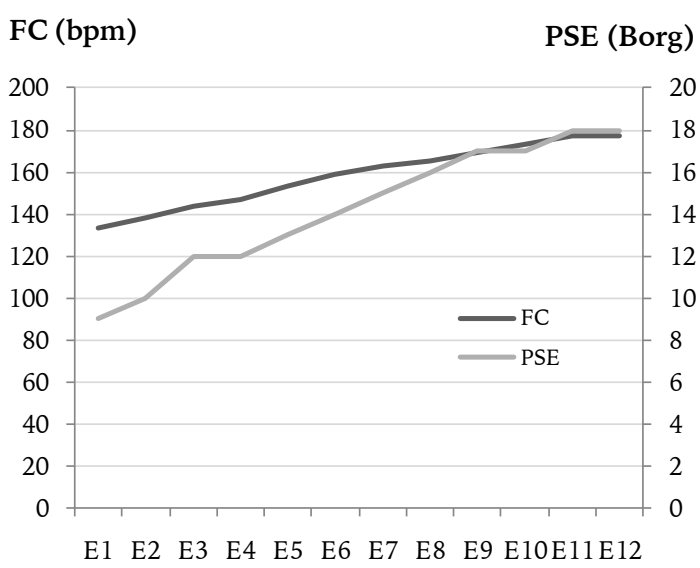

Figura 2. Médias por estágios da frequência cardíaca (FC) e percepção de esforço (PSE) obtidas pelo examinador $\mathrm{B}$

\section{DISCUSSÃO}

O principal achado deste estudo foi que as respostas obtidas para a FC e PSE no protocolo de esforço proposto foram reprodutíveis entre examinadores distintos. Esse fato pode ser verificado pelos altos valores de correlação encontrados e, em média, houve similaridade estatística entre as medições dos avaliadores. Isso significa que independente do avaliador, os escores no protocolo empregado são consistentes de um avaliador para o outro (Morrow, Jackson, Disch, \& Mood, 2006).

À luz da literatura específica, poucas investigações avaliaram a reprodutibilidade interavaliador ou teste-reteste dos protocolos de DWR. Mercer e Jensen (1997) analisaram a reprodutibilidade e validade de um protocolo de corrida em piscina funda em uma amostra de homens e mulheres adultos, encontrando similaridade para as respostas do $\mathrm{VO}_{2}$ pico e FC entre sucessivos testes de DWR $(p>.05)$, além de boas correlações $(.74-.94)$. Estudos comparativos da corrida em meio aquático versus terrestre são comuns (Killgore, Wilcox, Caster, \& Wood, 2006; Phillips, Legge \& Jones, 2008), entretanto a riqueza de detalhes 
apresentadas por estágio em nosso estudo é peculiar.

A variabilidade da PSE encontrada nos estágios intermediários (E5, E7, E8 e E9) (ver tabela 3) pode ser atribuída ao desempenho irregular de muitos sujeitos entre as medidas efetuadas pelos avaliadores. Embora as médias de FC nestes estágios não acompanharem essa tendência, alerta-se para a necessidade de um treinamento na aplicação do protocolo para os avaliadores, especialmente nos estágios destacados.

Constatou-se que tanto a PSE quanto a FC aumentaram linearmente em relação ao incremento das cadências durante o exercício de corrida em piscina funda, sendo a correlação entre estas variáveis mais evidente para uma maior intensidade. Ikuo, Tomo e Shigeru (1999) encontraram resultados semelhantes ao verificarem que a PSE se comportou de forma proporcional e linear em relação às respostas cardiorrespiratórias durante corrida em piscina funda no protocolo de incremento da intensidade, justificando os nossos achados.

$\mathrm{O}$ aumento do número de indivíduos que ultrapassaram os estágios da primeira execução do protocolo, traduzido por melhoras entre tentativas repetidas, é reportado na literatura no que se refere a um desempenho significativamente melhor em sua segunda tentativa devido à adaptação sofrida pelo indivíduo (Coelho et al., 2007).

Com relação às respostas de FC e PSE, Shono, Fujishima, Hotta, Ogaki e Ueda (2000) examinaram estas variáveis durante caminhada em esteira dentro de água e mostraram forte relação linear $(r=.99, p<.01)$, concluindo assim que a PSE pode ser considerada um bom índice para prescrição de caminhada em esteira, tanto na água, quanto no meio terrestre.

Ao analisar a relação entre as cadências e a FC durante a corrida em piscina funda, Wilder et al. (1993) encontraram uma correlação de .73, apresentando um comportamento linear a cada incremento, e para correlação intraindividual um $r=.98$. Estas pesquisas suportam os achados do presente estudo no que se refere à relação entre as medidas de FC e de PSE.

O protocolo utilizado representa uma inovação na forma de controlar a cadência em relação a outros trabalhos (Kaminsky et al., 1993; Sherman \& Michaud, 1997), pelo fato de orientar os sujeitos por meio de bips.

Entretanto, o protocolo possui limitações no tocante à dificuldade de visualização da execução do gesto motor devido à refração da água, uma vez que este é um dos critérios para a detecção da falha na execução do movimento. Além disso, o colete flutuador utilizado não apresenta uma individualização, o que pode permitir uma maior proporção entre a massa corporal do indivíduo e a massa do colete.

A partir dessas considerações, verifica-se que este protocolo pode ser aplicado por profissionais em situações em que haja necessidade de avaliar melhorias específicas da condição cardiovascular nas atividades aquáticas em geral.

\section{CONCLUSÕES}

O protocolo de DWR, que se utilizou de bips para orientar a cadência do exercício na água, foi reprodutível quando aplicado por avaliadores distintos.

Dessa forma, obtiveram-se respostas similares nas medidas de FC e PSE com o incremento da intensidade para a amostra investigada. Os resultados analisados por meio de correlações e testes de hipótese podem prover evidências para a validação do protocolo de DWR empregado, indicando que o mesmo possui aplicação profissional. Correlações mais evidentes entre a FC e a PSE foram encontradas a partir do oitavo estágio, quando a intensidade do exercício é manifestamente mais elevada.

Este achado infere sobre a melhoria da prescrição e monitoramento da intensidade em atividades aquáticas, em especial para a corrida em piscina funda, visto que estas variáveis aumentaram linearmente em relação ao incremento da carga. 


\section{REFERÊNCIAS}

Alves, R. V., Mota J., Costa M. C., \& Alves, J. G. B. (2004). Fitness and elderly health effects of hydrogymnastics. Revista Brasileira de Medicina do Esporte, 10(1), 38-43.

Borg, G. (2000). Escalas de Borg para a dor e o esforço percebido. São Paulo: Manole.

Broman, G., Quintana, M., Lindberg, T., Jansson, E., \& Kaijser, L. (2006). High intensity deep-water training can improve aerobic power in elderly women. European Journal of Applied Physiology, 98(2), 117-123.

Coelho, C. C., Aquino, E. S., Almeida, D. C., Oliveira, G. C., Pinto, R. C., Rezende, I. M. O., et al. (2007). Análise comparativa e reprodutibilidade do teste de caminhada com carga progressiva (modificado) em crianças normais e em portadoras de fibrose cística. Jornal Brasileiro de Pneumologia, 33 (2), 168-174.

Craig, C. L., Marshall, A. L., Sjöström, M., Bauman, A. E., Booth, M. L., Ainsworth, B. E., et al. (2003). International physical activity questionnaire: 12-country reliability and validity. Medicine $\mathcal{E}$ Science in Sports \& Exercise, 35(8), 1381-1395.

Dolbow, D. R., Farley, R. S., Kim, J. K., \& Caputo, J. L. (2008). Oxygen consumption, heart rate, rating of perceived exertion, and systolic blood pressure with water treadmill walking. Journal of Aging and Physical Activity, 16, 14-23.

Green, J. H., Cable, N. T., \& Elms, N. (1990). Heart rate and oxygen consumption during walking on land and in deep water. Journal of Sports Medicine and Physical Fitness, 30(1), 49-52.

Ikuo, T., Tomo, O., \& Shigeru, O. (1999). The physiological responses and RPE to different water flow and belt velocities during "flow mill" walking in water. Journal of Health and Sports Science, 3, 61-67.

International Society for the Advancement of Kinantropometry (ISAK) (2000). International standards for anthropometric assessment. Australia: Autor.

Kaminsky, L. A., Wehrli, K. W., Mahon, A. D., Robbins, G. C., Powers, D. L., \& Whaley, M. H. (1993). Evaluation of a shallow waterrunning test for the estimation of peak aerobic power. Medicine $\mathcal{E}$ Science in Sports $\mathcal{E}$ Exercise, 25(11), 1287-1292.

Kilding, A. E., Scott, M. A., \& Mullineaux, D. R. (2007). A kinematic comparison of deep-water running and overground running in endurance runners. Journal of Strength and Conditioning Research, 21 (2), 476-480.

Killgore, G., Wilcox, A., Caster, B., \& Wood, T. (2006). A lower extremities kinematic comparison of deep-water running styles and treadmill running. Journal of Strength and Conditioning Research, 20(4), 919-927,

Lindle, M. J., Wasserman, F. J., \& See, L. J. (2001). Manual do profissional de fitness aquático (AEA). Rio de Janeiro: Shape.

McGhee, D. E., Power, B. M., \& Steele J. R. (2007). Buoyancy and breast bounce: Does deep-water running reduce exercise-induced breast discomfort? British Journal of Sports Medicine, 41 (12), 879-883.

Mercer, J. A., \& Jensen, R.L. (1997). Reliability and validity of a deep-water running graded exercise test. Measurement in Physical Education and Exercise Science, 1 (4), 213-222.

Michaud, T. J., Rodriguez-Zayas, J., Andres, F. F., Flynn, M. G., \& Lambert, C. P. (1995). Comparative exercise responses of deep-water and treadmill running. Journal of Strength and Conditioning Research, 9(2), 104-109.

Morrow, J., Jackson, A., Disch, J., \& Mood, D. (2006). Measurement and evaluation in human performance ( $3^{\mathrm{a}}$ ed). Champaign, IL: Human Kinetics Publishers.

Nelson, M. E., Rejeski, W. J., Blair S. N., Duncan, P. W., Judge, J. O., King, A. C., et al. (2007). Physical activity and public health in older adults: Recommendation from the American College of Sports Medicine and the American Heart Association. Medicine $\mathcal{E}$ Science in Sports \& Exercise, 39(8), 1423-1434.

Pantoja, P. D., Vendrusculo, A. P., Fayh, A. P., Alberton, C. L., \& Kruel, L. F. M. (2006). Respostas hemodinâmicas, cardiorrespiratórias e ocorrência de lesão muscular no meio aquático e terrestre em mulher não ativa: Estudo de caso. Motriz, 12(3), 271-276.

Phillips, V. K., Legge M., \& Jones, L. M. (2008). Maximal physiological responses between aquatic and land exercise in overweight women. Medicine $\mathcal{E}$ Science in Sports $\mathcal{E}$ Exercise, 40(5), 959-964.

Pollock, G. A. G., Butcher, J., Després, J., Dishman, R. K., Franklin, B. A., \& Garber, C. E. (1998). The recommended quantity and quality of exercise for developing and maintaining cardiorespiratory and muscular fitness, and 
54 | I.R.S. Silva, L.S. Oliveira, M.F. Berenguer, A.V.F. Sousa, J.A. Nascimento, M.C. Costa

flexibility in healthy adults. Medicine $\mathcal{E}$ Science in Sports \& Exercise, 30(6), 975-991.

Sherman, N. W., \& Michaud, T. J. (1997). $\mathrm{VO}_{2} \max$ estimation in healthy adults using submaximal deep-water running. Journal of Strength and Conditioning Research, 11 (2), 73-76.

Shono, T., Fujishima, K., Hotta, N., Ogaki, T., \& Ueda, T. (2000). Physiological responses to water-walking in middle aged women. Journal of Physiological Anthropology and Applied Human Science, 20(2), 119-123.

Wilder, R. P., Brennan, D. K., \& Schotte, D. E. (1993). A standard measure for exercise prescription for aqua running. American Journal of Sports Medicine, 21 (8), 45-48. 